\title{
Controlo intensivo da pressão arterial na diabetes mellitus tipo 2: qual a evidência?
}

Vânia Gomes, ${ }^{1}$ Sara Domingues ${ }^{2}$

\section{RESUMO}

Objetivo: Verificar se o efeito do tratamento anti-hipertensor intensivo (pressão arterial sistólica $<130 \mathrm{mmHg}$ ) apresenta benefícios na morbimortalidade cardiovascular em doentes com diabetes mellitus tipo 2 e com hipertensão arterial, em comparação com o tratamento anti-hipertensor standard (pressão arterial sistólica $<140 \mathrm{mmHg}$ ).

Fontes de dados: National Guideline Clearinghouse, NHS Evidence, CMA InfoBase, Cochrane, DARE, MEDLINE/PubMed.

Métodos de revisão: Pesquisa de meta-análises (MA), revisões sistemáticas, ensaios clínicos controlados randomizados (ECR) e guidelines, nas línguas inglesa e portuguesa, publicados entre 2006 e 2016, com os termos MeSH: antihypertensive agents, diabetes mellitus e blood pressure. Foi aplicada a escala Strength of Recommendation Taxonomy (SORT), da American Family Physician, para avaliação dos níveis de evidência (NE) e da força de recomendação (FR).

Resultados: Obtiveram-se 662 artigos e, destes, 12 cumpriram os critérios de inclusão: três MA, um ECR e oito guidelines. As MA revelaram que o controlo intensivo da pressão arterial (PA) em doentes diabéticos diminui o risco de acidente vascular cerebral, de microalbuminúria e de nefropatia (NE 2). Contudo, o controlo intensivo da PA nestes doentes associa-se a maior risco de efeitos adversos e parece aumentar a mortalidade cardiovascular (NE 2). O ECR demonstrou apenas redução do risco de microalbuminúria (NE 2). Por fim, a maioria das guidelines não recomenda um controlo intensivo da PA em doentes diabéticos e considera que o controlo da PA deve ser mais exigente apenas em diabéticos jovens, com lesão de órgão-alvo ou com mais do que um fator de risco para doença cardiovascular aterosclerótica.

Conclusões: Perante a evidência disponível, o controlo intensivo da PA em doentes com diabetes mellitus tipo 2 não deve ser recomendado, exceto em diabéticos jovens e em determinados grupos de risco (FR B). Porém, este estudo reforça a importância do controlo da PA nestes doentes, alertando para o papel fundamental do médico de família na avaliação individualizada dos riscos e benefícios do tratamento intensivo da PA.

Palavras-chave: Hipertensão; Diabetes mellitus tipo 2; Agentes anti-hipertensores.

\section{INTRODUÇÃO}

A diabetes mellitus (DM) tipo 2 e a hipertensão arterial (HTA) são fatores de risco prevalentes e com elevado impacto mundial. A prevalência global da DM ronda os $8,5 \%$, correspondendo a maioria a doentes com DM tipo 2, e tem vindo a aumentar nas últimas décadas. ${ }^{1}$ A DM tipo 2 é um dos fatores de risco major para eventos cardiovasculares, como o enfarte agudo do miocárdio (EAM) e o acidente vascular cerebral (AVC), e está associada a várias complicações microvasculares,

1. Médica Interna de Medicina Geral e Familiar. USF Santo António, ACeS Cávado III -Barcelos/Esposende.

2. Médica Interna de Medicina Geral e Familiar. USF Pró-Saúde, ACeS Cávado II-Gerês/Cabreira. nomeadamente, a nefropatia, a retinopatia e a neuropatia. ${ }^{1-2}$ As complicações cardiovasculares constituem a principal causa de morte, sendo responsáveis por $70 \%$ das mortes nestes doentes. ${ }^{2-3}$ Além disso, a HTA é uma comorbilidade presente em $75 \%$ dos doentes diabéticos e a sua associação com a DM tipo 2 aumenta em $57 \%$ o risco de eventos cardiovasculares..$^{4-6} \mathrm{~A}$ coexistência destas duas patologias também aumenta o risco de insuficiência cardíaca (IC), nefropatia e outros eventos microvasculares. ${ }^{6}$ Assim, o controlo da pressão arterial (PA) nos doentes diabéticos é um pilar fundamental na prática clínica.

Neste contexto, vários estudos clínicos controlados e randomizados revelaram, inequivocamente, que a 
redução da PA é a intervenção mais efetiva para a redução da morbimortalidade cardiovascular nos doentes com DM tipo 2.5,7-8 Até recentemente, as guidelines internacionais recomendavam universalmente que a HTA em doentes diabéticos devia ter como alvo terapêutico valores inferiores a 130/80 $\mathrm{mmHg} \cdot{ }^{9 \cdot 11}$ Contudo, novas evidências sugerem que uma pressão arterial sistólica (PAS) mais baixa pode estar associada a um aumento do risco cardiovascular, além de um aumento do risco de efeitos adversos. ${ }^{12-13}$ Neste sentido, atualmente a maioria das guidelines recomenda valores de PA inferiores a $140 / 90 \mathrm{mmHg}$ como valor alvo de PA em doentes diabéticos. ${ }^{14-16}$

Deste modo, até ao momento o valor de pressão arterial ideal nos doentes com DM tipo 2 ainda é incerto e objeto de grande discussão.

O objetivo desta revisão é verificar se o efeito do tratamento anti-hipertensor intensivo (PAS $<130 \mathrm{mmHg}$ ) apresenta benefícios na morbimortalidade cardiovascular, em doentes com DM tipo 2 e com HTA, em comparação com o tratamento anti-hipertensor standard (PAS $<140 \mathrm{mmHg}$ ).

\section{MÉTODOS}

Foi realizada uma pesquisa de meta-análises (MA), revisões sistemáticas, ensaios clínicos controlados randomizados (ECR) e guidelines, com os termos MeSH antihypertensive agents, diabetes mellitus e blood pressure, nas bases de dados National Guideline Clearinghouse, NHS Evidence, CMA infobase, The Cochrane Library, DARE, MEDLINE/PubMed. Incluíram-se os artigos publicados entre janeiro de 2006 e maio de 2016, nas línguas inglesa e portuguesa, que respeitassem os seguintes critérios de elegibilidade:

- População: indivíduos adultos com DM tipo 2 e com HTA;

- Intervenção: tratamento anti-hipertensor intensivo (PAS <130mmHg);

- Comparação: tratamento anti-hipertensor standard (PAS $<140 \mathrm{mmHg}$ );

- Outcome (orientado para o doente): morbilidade (complicações macrovasculares e microvasculares) e mortalidade cardiovascular.

Considerou-se como alvo terapêutico apenas a PAS, uma vez que, por um lado, esta é um preditor constante de eventos cardiovasculares e, por outro lado, a PA diastólica parece assumir maior importância como preditor de eventos cardiovasculares apenas em indivíduos com idade inferior a 60 anos. ${ }^{17}$ Definiu-se como tratamento anti-hipertensor intensivo o atingimento de uma PAS inferior a 130 $\mathrm{mmHg}$ porque se trata do alvo terapêutico de PAS para indivíduos diabéticos recomendado nas guidelines mais antigas. ${ }^{9-11}$

Foram excluídos estudos que compararam os efeitos de diferentes fármacos anti-hipertensores, que avaliaram os outcomes sem estratificar para os valores alvo de PA e estudos já incluídos nas meta-análises e artigos de opinião.

Para avaliar os níveis de evidência (NE) e atribuir graus de forças de recomendação (FR) foi aplicada a escala Strenght of Recommendation Taxonomy (SORT), da American Family Physician.

\section{RESULTADOS}

Na pesquisa bibliográfica inicial obtiveram-se 662 artigos, dos quais 12 cumpriram os critérios de inclusão: três MA, um ECR e oito guidelines. O processo de seleção dos artigos está descrito no fluxograma da Figura 1 e os resultados encontram-se sumariados nos Quadros I, II e III.

A meta-análise de Brunström e colaboradores, ${ }^{12} \mathrm{pu}-$ blicada em 2016, teve como objetivo avaliar o efeito do tratamento anti-hipertensor na mortalidade e morbilidade cardiovascular em indivíduos com DM tipo 2. Os autores estratificaram os resultados para diferentes níveis de PAS basal e PAS atingida após tratamento: PAS basal $>150 \mathrm{mmHg}$ vs $150-140 \mathrm{mmHg} v s<140 \mathrm{mmHg}$; PAS atingida após tratamento $>140 \mathrm{mmHg} v s$ 140-130mmHg $v s<130 \mathrm{mmHg}$. Verificaram que o tratamento anti-hipertensor reduz o risco de mortalidade total nos indivíduos com DM tipo 2 com PAS basal $>140 \mathrm{mmHg}$ e PAS atingida entre 140 e 130mmHg. Contudo, nos diabéticos tipo 2 com PAS basal $<140 \mathrm{mmHg}$ o tratamento esteve associado a aumento do risco de morte cardiovascular, o que também se verificou, sem significado estatístico, para o grupo com PAS atingida $<130 \mathrm{mmHg}$. Constataram ainda uma diminuição do risco de EAM e de IC nos diabéticos com PAS basal >140mmHg e com PAS atingida $>130 \mathrm{mmHg}$, bem como uma diminuição do risco de AVC nos diabéticos com PAS basal $>140 \mathrm{mmHg}$ e PAS atingida $<140 \mathrm{mmHg}$. Por fim, o único subgrupo que demonstrou diminuição no risco de 


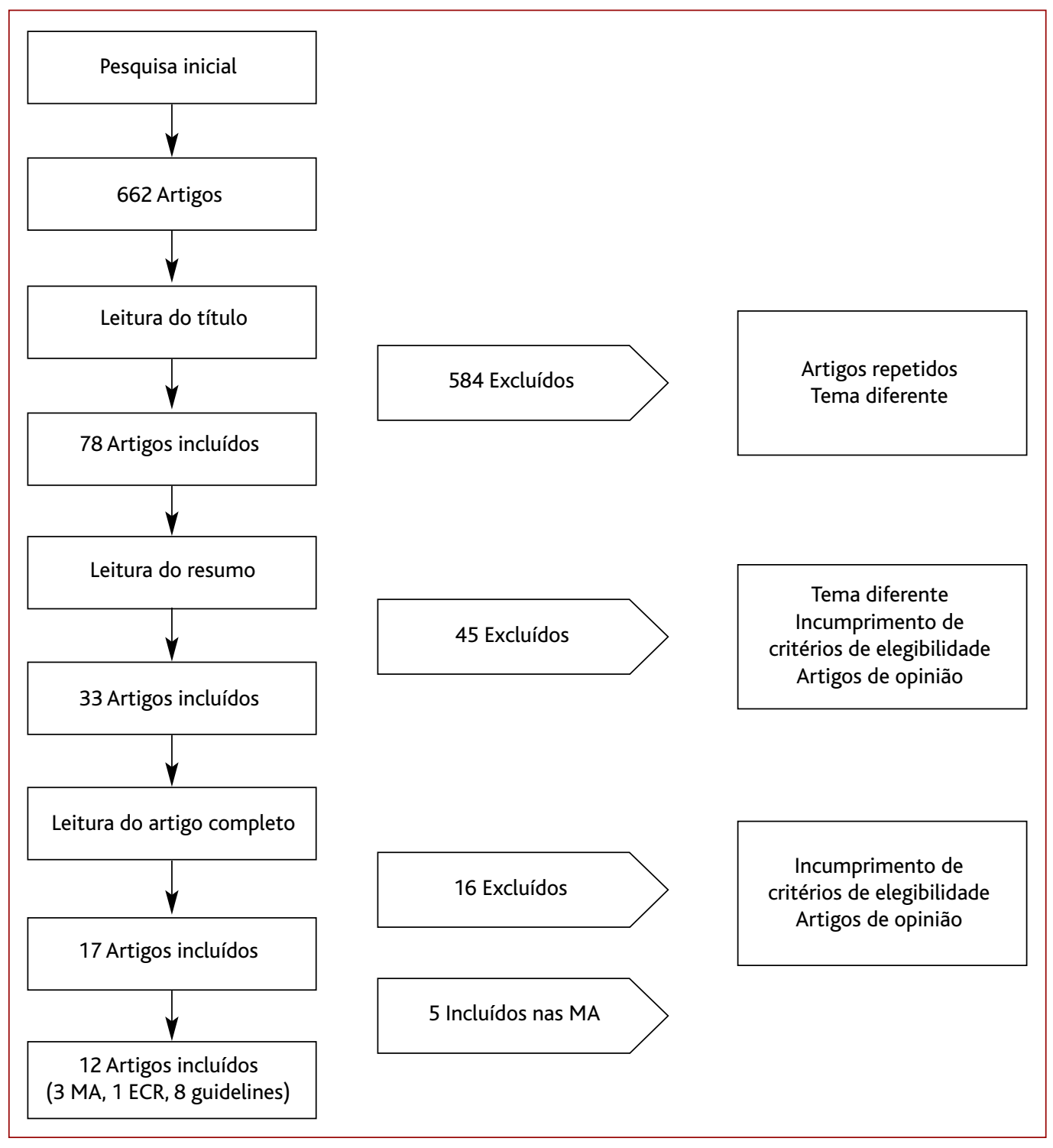

Figura 1. Fluxograma da seleção dos artigos.

Legenda: $M A$ = Meta-análise; $E C R$ = Ensaios clínicos controlados randomizados.
ECR individualmente; utilização de variável agregada, ou seja, média dos valores de PAS basal e atingida nos diferentes ECR, o que possibilita a ocorrência do viés ecológico; variabilidade nos outcomes reportados pelos vários ECR; outcomes amputação e cegueira não foram analisados porque eram incluídos em poucos ECR; e utilização de diferentes classes de medicamentos no tratamento da HTA, nomeadamente uso de esquemas terapêuticos não recomendados atualmente (dupla inibição do eixo renina-angiotensina).

A meta-análise de Emdin e colaboradores, ${ }^{18}$ publicada em 2015, teve como objetivo determinar se a dimi- doença renal terminal foi o subgrupo com PAS basal $>150 \mathrm{mmHg}$. Assim, sugerem que o alvo do tratamento da tensão arterial nestes doentes deve ser menos agressivo, uma vez que o efeito do tratamento é menos benéfico para PAS mais baixas. Todavia, esta meta-análise apresenta algumas limitações: a maioria dos ECR não foi desenhada para comparar alvos de PAS, mas para comparar eficácia de fármaco comparativamente ao placebo; inclui todos os ECR com follow-up médio de 12 meses, não especificando o follow-up de cada nuição da PA em indivíduos com DM tipo 2 está associada a diminuição do risco de eventos macro e microvasculares. Para isso, os autores estratificaram os participantes em dois grupos de acordo com a PAS basal antes do tratamento e a PAS atingida após o tratamento. Em cada um dos grupos os participantes foram subdivididos em dois subgrupos - PAS basal $\geq 140 \mathrm{mmHg} v s$ $<140 \mathrm{mmHg}$ e PAS após o tratamento $\geq 130 \mathrm{mmHg} v s$ $<130 \mathrm{mmHg}$. Numa análise global verificaram que a diminuição de $10 \mathrm{mmHg}$ da PAS em indivíduos diabéti- 


\section{QUADRO I. Meta-análises}

\begin{tabular}{|c|c|c|c|c|}
\hline $\begin{array}{l}\text { Referência } \\
\text { (ano) }\end{array}$ & Metodologia & Resultados & Limitações & $\mathrm{NE}^{*}$ \\
\hline $\begin{array}{l}\text { Brunström, } \\
\text { et al } \\
(2016)^{12}\end{array}$ & $\begin{array}{l}49 \text { ECR } \\
N=73.738 \\
\text { Follow-up médio: } 3,7 \text { anos } \\
\text { Comparação entre grupos de } \\
\text { doentes com diabetes mellitus } \\
\text { tipo 2: } \\
\text { - PAS basal: }>150 \mathrm{mmHg} v \mathrm{~s} \\
\text { 150-140mmHg vs }<140 \mathrm{mmHg} \\
\text { - PAS atingida após tratamento } \\
\text { anti-hipertensor: }>140 \mathrm{mmHg} v \text { s } \\
\text { 140-130mmHg vs }<130 \mathrm{mmHg} \\
\text { Complicações macrovasculares } \\
\text { Outcomes: mortalidade total, } \\
\text { mortalidade CV, EAM, AVC, IC. } \\
\text { Complicações microvasculares } \\
\text { Outcomes: doença renal terminal. }\end{array}$ & 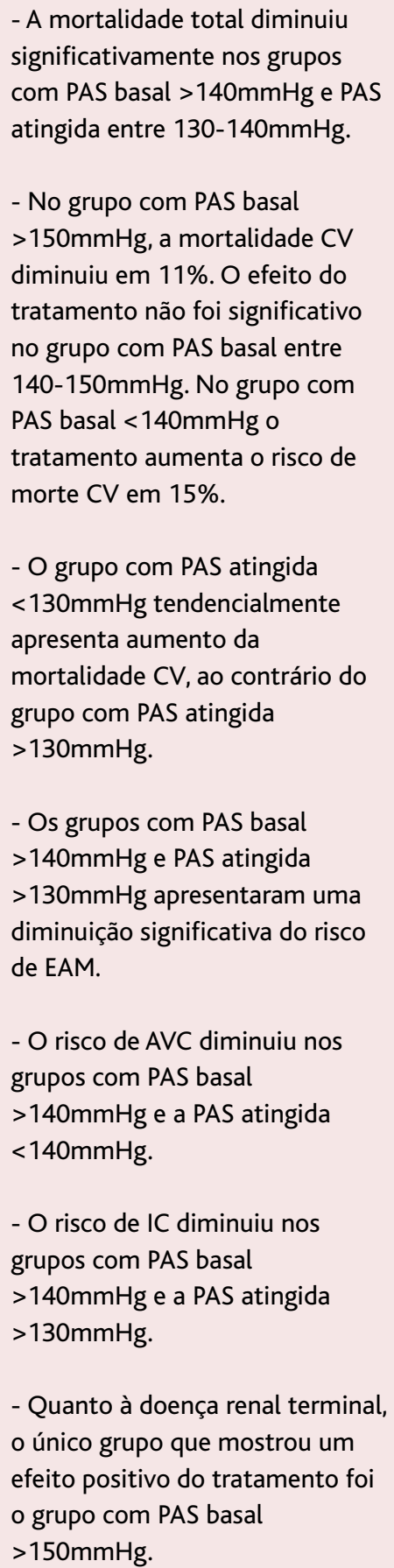 & $\begin{array}{l}\text { - Maioria dos ECR não foi } \\
\text { desenhado para comparar alvos } \\
\text { de PAS. } \\
\text { - Inclui todos os ECR com } \\
\text { follow-up médio de } 12 \text { meses, } \\
\text { não especificando o follow-up de } \\
\text { cada ECR individualmente nem o } \\
\text { desvio-padrão da média dos } \\
\text { tempos de follow-up. } \\
\text { - Viés ecológico. } \\
\text { - Variabilidade nos outcomes } \\
\text { reportados pelos vários ECR. } \\
\text { - Outcomes amputação e } \\
\text { cegueira não foram analisados. } \\
\text { - Utilização de diferentes classes } \\
\text { de medicamentos no tratamento } \\
\text { da HTA. }\end{array}$ & 2 \\
\hline
\end{tabular}

\footnotetext{
* Nível de evidência atribuído pelas autoras.
} 


\begin{tabular}{|c|c|c|c|c|}
\hline $\begin{array}{l}\text { Referência } \\
\text { (ano) }\end{array}$ & Metodologia & Resultados & Limitações & NE* \\
\hline $\begin{array}{l}\text { Emdin, et al } \\
(2015)^{18}\end{array}$ & $\begin{array}{l}45 \text { ECR } \\
N=104.586 \\
\text { Comparação entre grupos de } \\
\text { doentes com diabetes mellitus } \\
\text { tipo 2: } \\
\text { - PAS basal: } \geq 140 \mathrm{mmHg} \text { vs } \\
<140 \mathrm{mmHg} \\
\text { - PAS atingida após tratamento } \\
\text { anti-hipertensor: } \geq 130 \mathrm{mmHg} \text { vs } \\
<130 \mathrm{mmHg} \\
\text { Complicações macrovasculares } \\
\text { Outcomes: mortalidade total, } \\
\text { eventos CV, doença coronária, IC, } \\
\text { AVC. } \\
\text { Complicações microvasculares } \\
\text { Outcomes: albuminúria. }\end{array}$ & $\begin{array}{l}\text { - A diminuição de } 10 \mathrm{mmHg} \text { da } \\
\text { PAS nos indivíduos com PAS basal } \\
\geq 140 \mathrm{mmHg} \text { ou com PAS atingida } \\
\geq 130 \mathrm{mmHg} \text { esteve associada a } \\
\text { diminuição significativa da } \\
\text { mortalidade total, de eventos CV, } \\
\text { de doença coronária e de IC. } \\
\text { - A diminuição da PAS diminuiu o } \\
\text { risco de AVC, independentemente } \\
\text { do valor basal de PAS ou do valor } \\
\text { da PAS atingido. } \\
\text { - A diminuição de PA diminuiu o } \\
\text { risco de albuminúria, } \\
\text { independentemente do valor } \\
\text { basal da PAS ou do valor da PAS } \\
\text { atingida. }\end{array}$ & $\begin{array}{l}\text { - Maioria dos ECR não foi } \\
\text { desenhada para comparar alvos } \\
\text { de PAS. } \\
\text { - Escassez de ECR com nível de } \\
\text { PAS atingida entre } 120 \text { e } \\
\text { 130mmHg. } \\
\text { - Utilização de diferentes classes } \\
\text { de medicamentos no tratamento } \\
\text { da HTA. } \\
\text { - Reduzido tempo de follow-up. }\end{array}$ & 2 \\
\hline $\begin{array}{l}\text { Bangalore, } \\
\text { et al } \\
(2011)^{13}\end{array}$ & $\begin{array}{l}13 \text { ECR } \\
\text { N=37.763 } \\
\text { Follow up: } 4,8 \pm 1,3 \text { anos } \\
\text { Comparação entre } 2 \text { grupos de } \\
\text { doentes com diabetes mellitus } \\
\text { tipo 2: } \\
\text { Grupo submetido a tratamento } \\
\text { anti-hipertensor intensivo (PAS } \\
\text { atingida } \leq 135 \text { mmHg; subgrupo } \\
\leq 130 \text { mmHg): } \\
\text { 19.042 participantes (50,5\%) } \\
\text { vs } \\
\text { Grupo submetido a tratamento } \\
\text { anti-hipertensor standard (PAS } \\
\text { atingida } \leq 140 \text { mmHg): } \\
\text { 18.694 participantes (49,5\%) }\end{array}$ & $\begin{array}{l}\text { - O grupo com controlo intensivo } \\
\text { da PA apresentou uma diminuição } \\
\text { significativa de } 10 \% \text { na } \\
\text { mortalidade total, com maior } \\
\text { contribuição do subgrupo com } \\
\text { PAS entre } 130-135 \mathrm{mmHg} \text {. } \\
\text { - O controlo intensivo da PA } \\
\text { diminui em } 17 \% \text { o risco de AVC. } \\
\text { Nos estudos em que a PAS alvo } \\
\text { foi } \leq 130 \mathrm{mmHg} \text { ocorreu uma } \\
\text { redução significativa do risco de } \\
\text { AVC em } 47 \% \text {. } \\
\text { - O grupo com controlo intensivo } \\
\text { da PA apresentou uma diminuição } \\
\text { de } 17 \% \text { no risco de desenvolver } \\
\text { microalbuminúria. }\end{array}$ & $\begin{array}{l}\text { - Variabilidade nos outcomes } \\
\text { reportados pelos vários ECR. } \\
\text { - Utilização do IECA como } \\
\text { anti-hipertensor em alguns } \\
\text { estudos. } \\
\text { - Influência da sobrevivência dos } \\
\text { indivíduos na PAS alcançada. } \\
\text { - Diferentes definições atribuídas } \\
\text { ao conceito «efeitos adversos } \\
\text { severos». }\end{array}$ & 2 \\
\hline
\end{tabular}

cos contribui para uma diminuição significativa do risco de mortalidade global, de eventos cardiovasculares e de AVC. Contribui também, embora de forma não significativa, para diminuição do risco de retinopatia e de albuminúria. Numa análise estratificada verificaram que a diminuição de $10 \mathrm{mmHg}$ da PAS em indiví- 


\section{QUADRO I. Meta-análises (continuação)}

\begin{tabular}{|c|c|c|c|c|}
\hline $\begin{array}{l}\text { Referência } \\
\text { (ano) }\end{array}$ & Metodologia & Resultados & Limitações & NE* \\
\hline & $\begin{array}{l}\text { Complicações macrovasculares } \\
\text { Outcomes: mortalidade total, } \\
\text { mortalidade CV, EAM, AVC, IC, } \\
\text { angina, revascularização. } \\
\text { Complicações microvasculares } \\
\text { Outcomes: microalbuminúria, } \\
\text { nefropatia, doença renal } \\
\text { terminal/diálise, neuropatia, } \\
\text { retinopatia. } \\
\text { Efeitos adversos severos } \\
\text { Outcomes: eventos life- } \\
\text { threatening, eventos que causam } \\
\text { incapacidade permanente, } \\
\text { eventos com necessidade de } \\
\text { hospitalização. }\end{array}$ & $\begin{array}{l}\text { - O grupo com controlo intensivo } \\
\text { de PA apresentou menor risco de } \\
\text { nefropatia. Este benefício foi } \\
\text { superior nos estudos com alvos } \\
\text { de PAS } \leq 130 \mathrm{mmHg} \text {, verificando-se } \\
\text { uma redução de } 36 \% \text {. } \\
\text { - Os dois grupos não apresentaram } \\
\text { diferenças na mortalidade } \\
\text { cardiovascular, no risco de EAM, } \\
\text { de IC, de angina, de doença renal } \\
\text { terminal/diálise, de retinopatia, de } \\
\text { neuropatia e na necessidade de } \\
\text { revascularização. } \\
\text { - Nos estudos com PAS alvo } \\
\leq 130 m m H g \text { verificou-se um } \\
\text { aumento de } 40 \% \text { no risco de } \\
\text { efeitos adversos severos. }\end{array}$ & & \\
\hline
\end{tabular}

duos diabéticos com PAS basal $\geq 140 \mathrm{mmHg}$ ou com PAS atingida $\geq 130 \mathrm{mmHg}$ esteve associada a diminuição significativa da mortalidade global, de doença cardiovascular e de IC. Estes resultados não se revelaram significativos no grupo de indivíduos com PAS basal $<140 \mathrm{mmHg}$ ou PAS atingida $<130 \mathrm{mmHg}$. Por fim, a diminuição da PAS diminui o risco de AVC e o risco de albuminúria, independentemente do valor basal da PAS ou do valor da PAS atingida. Esta meta-análise apresenta, contudo, algumas limitações: a maioria dos ECR não foi desenhada para comparar alvos de PAS, mas para comparar eficácia de fármaco comparativamente ao placebo; a escassez de ensaios clínicos com nível de PAS atingida entre 120 e 130mmHg; a utilização de diferentes classes de medicamentos no tratamento da HTA, o que poderá influenciar a eficácia do tratamento e os eventos adversos; e o reduzido tempo de follow-up dos ECR, o que poderá ter impossibilitado a associação entre a diminuição da PA e os outcomes vasculares, particularmente a insuficiência cardíaca e a insuficiência renal.

A meta-análise de Bangalore e colaboradores, ${ }^{13} \mathrm{pu}$ - blicada em 2011, teve como objetivo avaliar o valor alvo da pressão arterial em indivíduos com DM tipo 2. Para isso, os autores analisaram e estratificaram as complicações macro e microvasculares a longo prazo e os efeitos adversos severos em dois grupos: o grupo submetido a tratamento anti-hipertensor intensivo com o valor alvo de PAS $\leq 135 \mathrm{mmHg}$ e o grupo submetido a tratamento anti-hipertensor standard com o valor alvo de PAS $\leq 140 \mathrm{mmHg}$. O grupo com controlo intensivo da PAS foi subdividido em dois: o grupo com tratamento mais intensivo (PAS $\leq 130 \mathrm{mmHg}$ ) e o grupo com tratamento menos intensivo (PAS entre 130 e $135 \mathrm{mmHg}$ ). No que concerne às complicações macrovasculares, o controlo intensivo da PAS $(\leq 135 \mathrm{mmHg}$ ) reduz de forma significativa a mortalidade CV e risco de AVC. A diminuição da mortalidade CV foi mais evidente no subgrupo com PAS alvo entre 130 e 135mmHg, enquanto a diminuição do risco de AVC apresentou maior contribuição do subgrupo com PAS alvo $\leq 130 \mathrm{mmHg}$. No que diz respeito às complicações microvasculares, concluíram que o grupo com controlo intensivo da PA apresenta menor risco de microalbuminúria e de nefropatia evidente, 


\begin{tabular}{|c|c|c|c|c|}
\hline $\begin{array}{l}\text { Referência } \\
\text { (ano) }\end{array}$ & Metodologia & Resultados & Limitações & NE* \\
\hline $\begin{array}{l}\text { Ismail- } \\
\text {-Beigi, et al } \\
(2012)^{19}\end{array}$ & $\begin{array}{l}N=4.726 \\
\text { Follow-up: } 4,7 \text { anos } \\
\text { Comparação entre dois grupos de } \\
\text { doentes com diabetes mellitus } \\
\text { tipo 2: } \\
\text { Grupo submetido a tratamento } \\
\text { anti-hipertensor intensivo } \\
\text { (PAS < 120mmHg) } \\
\text { vs } \\
\text { Grupo submetido a tratamento } \\
\text { anti-hipertensor standard } \\
\text { (PAS < 140mmHg) } \\
\text { Complicações microvasculares } \\
\text { Outcomes: Albuminúria, falência } \\
\text { renal com necessidade de diálise } \\
\text { ou transplante, retinopatia, } \\
\text { neuropatia. }\end{array}$ & $\begin{array}{l}\text { - O controlo intensivo da PA } \\
\text { diminuiu de forma significativa o } \\
\text { desenvolvimento de } \\
\text { microalbuminúria em } 16 \% \text {. O } \\
\text { efeito sobre a macroalbuminúria } \\
\text { não foi significativo. } \\
\text { - O controlo intensivo da PA não } \\
\text { apresentou benefício significativo } \\
\text { nos restantes outcomes. }\end{array}$ & $\begin{array}{l}\text { - Sem ocultação dos participantes } \\
\text { e dos investigadores } \\
\text { relativamente ao tratamento } \\
\text { efetuado. } \\
\text { - Pequeno número de eventos } \\
\text { observados. } \\
\text { - Reduzida duração e intensidade } \\
\text { do tratamento. } \\
\text { - Início tardio do tratamento. } \\
\text { - Presença de diabetes de longa } \\
\text { duração e de complicações } \\
\text { microvasculares na população } \\
\text { em estudo. }\end{array}$ & 2 \\
\hline
\end{tabular}

* Nível de evidência atribuído pelas autoras.

com maior benefício no subgrupo com PAS alvo $\leq 130 \mathrm{mmHg}$. Por fim, constataram que o grupo com controlo intensivo da PA apresenta maior risco de efeitos adversos severos, havendo um aumento de $40 \%$ do risco no subgrupo cujo alvo de PAS foi $\leq 130 \mathrm{mmHg}$. Esta meta-análise apresenta algumas limitações: variabilidade nos outcomes reportados pelos vários ECR; utilização do inibidor da enzima de conversão da angiotensina (IECA) como anti-hipertensor em alguns estudos, pelo que não se pode afirmar com convicção de que os benefícios obtidos se devam inteiramente ao valor alvo da PAS atingido; influência de alguns fatores, como a sobrevivência dos indivíduos ao longo dos estudos, no valor de PAS alcançada; e diferenças na definição de efeitos adversos severos entre os diferentes estudos.

O ensaio clínico controlado e randomizado de Ismail-Beigi e colaboradores, ${ }^{19}$ publicado em 2012, teve como objetivo avaliar o efeito do tratamento intensivo da PA nas complicações microvasculares em indivíduos diabéticos. Os autores revelaram que o controlo intensivo da PA não apresenta benefícios significativos nas complicações microvasculares, exceto no desenvolvimento de microalbuminúria. Verificaram que o controlo intensivo de PA diminui, de forma significativa, o desenvolvimento de microalbuminúria em $16 \%$. O efeito sobre a macroalbuminúria, pelo contrário, não se revelou significativo e foi apenas aparente nos indivíduos com microalbuminúria de base. Contudo, os autores admitem que a ausência de benefício do controlo intensivo da PA na redução das complicações microvasculares pode ter sido devida ao pequeno número de eventos observados, à inadequada duração e intensidade do tratamento, ao início tardio do tratamento e ao facto de a população em estudo ter diabetes de longa duração e, portanto, com complicações microvasculares prévias, condicionando a resposta ao controlo intensivo da PA. De salientar ainda, como limitação deste estudo, a ausência de dupla ocultação, uma vez que quer os participantes quer os investigadores tinham 


\section{QUADRO III. Normas de orientação clínica}

\begin{tabular}{|c|c|c|}
\hline Referência (ano) & Recomendações & $\mathrm{FR}^{*}$ \\
\hline $\begin{array}{l}\text { American Diabetes Association } \\
(2016)^{20}\end{array}$ & $\begin{array}{l}\text { - Os doentes com DM tipo } 2 \text { devem ter como alvo terapêutico valor de PAS } \\
<140 \mathrm{mmHg} \text {. } \\
\text { - Nos doentes diabéticos jovens e nos doentes com albuminúria ou com } \geq \text { um fator de } \\
\text { risco para doença cardiovascular aterosclerótica o alvo terapêutico é um valor de PAS } \\
<130 \mathrm{mmHg} \text {. }\end{array}$ & A \\
\hline $\begin{array}{l}\text { NICE guideline } \\
\text { Type } 2 \text { diabetes in adults: } \\
\text { management }(2015)^{16}\end{array}$ & $\begin{array}{l}\text { - Doentes com DM tipo } 2 \text { devem ser tratados com o objetivo terapêutico de PAS } \\
<140 \mathrm{mmHg} \text {. } \\
\text { - Nos doentes com dano renal, cerebrovascular ou retinopatia, o alvo terapêutico deve } \\
\text { ser PAS }<130 \mathrm{mmHg} \text {. }\end{array}$ & B \\
\hline $\begin{array}{l}\text { Evidence-based guideline for } \\
\text { the management of high blood } \\
\text { pressure in adults }(2014)^{15}\end{array}$ & $\begin{array}{l}\text { - Em doentes com HTA e DM tipo 2, o tratamento farmacológico deve ter como } \\
\text { objetivo uma PAS }<140 \mathrm{mmHg} \text {. }\end{array}$ & B \\
\hline $\begin{array}{l}\text { Health care guideline: diagnosis } \\
\text { and management of type } 2 \\
\text { diabetes mellitus in adults } \\
(2014)^{14}\end{array}$ & - Num doente com DM tipo 2, o alvo terapêutico deve ser uma PAS < 140mmHg. & A \\
\hline $\begin{array}{l}\text { ESH/ESC Guidelines for the } \\
\text { management of arterial } \\
\text { hypertension }(2013)^{21}\end{array}$ & $\begin{array}{l}\text { - Em doentes com DM tipo } 2 \text { é recomendado um objetivo terapêutico de PAS } \\
<140 \mathrm{mmHg} \text {. }\end{array}$ & $B$ \\
\hline $\begin{array}{l}\text { The } 2010 \text { Canadian } \\
\text { hypertension education } \\
\text { program recommendations } \\
\text { for the management of } \\
\text { hypertension }(2010)^{22}\end{array}$ & $\begin{array}{l}\text { - Os doentes com DM tipo } 2 \text { devem ser tratados com o objetivo terapêutico de PAS } \\
<130 \mathrm{mmHg} \text {. }\end{array}$ & B \\
\hline $\begin{array}{l}\text { Clinical Practice guideline on } \\
\text { type } 2 \text { diabetes }(2008)^{23}\end{array}$ & $\begin{array}{l}\text { - Os doentes com HTA e DM tipo } 2 \text {, sem nefropatia ou retinopatia, devem ser tratados } \\
\text { com objetivo terapêutico de PAS }<140 \mathrm{mmHg} \text {. }\end{array}$ & B \\
\hline $\begin{array}{l}\text { Clinical Practice guidelines on } \\
\text { arterial hypertension }(2007)^{24}\end{array}$ & $\begin{array}{l}\text { - Nos doentes com HTA essencial e DM tipo } 2 \text { sem nefropatia, o alvo terapêutico } \\
\text { recomendado é uma PAS }<140 \mathrm{mmHg} \text {. }\end{array}$ & B \\
\hline
\end{tabular}

* Força de Recomendação atribuída pelas autoras. A FR A foi atribuída às Normas de Orientação Clínica baseadas em estudos consistentes e de boa qualidade metodológica; a FR B foi atribuída às Normas de Orientação Clínica baseadas em estudos consistentes, mas de limitada qualidade metodológica.

conhecimento sobre o tratamento que estava a ser realizado.

As guidelines ${ }^{14-16,20-24}$ apresentaram resultados consistentes. A maioria recomenda um alvo terapêutico de PAS < 140mmHg em doentes com DM tipo 2 e considera que o controlo da PAS deve ser mais intensivo (PAS $<130 \mathrm{mmHg}$ ) em diabéticos jovens e em diabéticos com albuminúria, retinopatia ou com mais do que um fator de risco para doença CV aterosclerótica.

\section{CONCLUSÕES}

Esta revisão baseada na evidência (RBE) revelou que o controlo intensivo da PA em doentes com DM tipo 2 não deve ser recomendado, exceto em diabéticos jovens e em determinados grupos de risco (FR B). Por um lado, o controlo intensivo da PAS em doentes diabéticos permite diminuir o risco de acidente vascular cerebral, de microalbuminúria e de nefropatia. Por outro lado, este controlo intensivo está associado a maior risco de efei- 
tos adversos e parece aumentar a mortalidade cardiovascular. Além disso, a maioria das guidelines ${ }^{14-16,20-21,23-24}$ não recomenda um controlo intensivo da PA em doentes diabéticos e considera que o controlo da PA deve ser mais exigente apenas em diabéticos mais jovens, com lesão de órgão-alvo ou com mais do que um fator de risco para doença cardiovascular aterosclerótica.

Os estudos incluídos nesta RBE apresentam diferenças metodológicas, o que contribui para a heterogeneidade dos resultados. A variabilidade na definição de controlo intensivo da PA e a utilização de diferentes valores terapêuticos dificultou a análise comparativa dos outcomes e impossibilitou atribuir uma recomendação linear do valor alvo de PA. Além disso, os resultados podem ter sido influenciados pela ausência de uniformização na classe de anti-hipertensores utilizados, pela utilização de esquemas terapêuticos não recomendados atualmente e pela curta duração de follow-up de vários ECR. Por fim, a maioria dos estudos não estabeleceu um valor alvo de PAD e não considerou as características individuais dos pacientes.

Nos últimos anos, vários autores ${ }^{12-13,25-28}$ têm demonstrado preocupação em analisar a segurança do controlo intensivo da PA em diabéticos. Tem sido sugerido que valores mais baixos de PAS podem estar associados a um aumento dos efeitos adversos, incluindo hipotensão ortostática, agravada pela neuropatia autonómica associada, síncope e maior risco de eventos que causam incapacidade permanente ou necessidade de hospitalização. Além disso, o controlo intensivo da PA pode aumentar o risco de morbimortalidade cardiovascular, uma vez que a diminuição da PA leva a diminuição da perfusão miocárdica, a qual é muito dependente da PAS, e a diminuição da circulação coronária colateral. ${ }^{12-13,28}$ Por fim, o risco de efeitos adversos parece ser aproximadamente igual ao grau de benefício na redução de eventos cerebrovasculares. ${ }^{26}$ Neste sentido, a redução da PA em indivíduos diabéticos deve ser individualizada, tendo em conta os riscos e benefícios inerentes ao tratamento.

No sentido de colmatar as falhas da evidência atual são necessários estudos com uniformização do valor alvo da PA e estudos que explorem o efeito dos diferentes anti-hipertensores nos vários outcomes. As meta-análises incluídas ${ }^{12-13,18}$ demonstraram que o valor basal da PA antes de iniciar o tratamento influencia e mo- difica o efeito do controlo intensivo da PA, pelo que estudos futuros deverão realizar uma análise estratificada, tendo em conta o valor basal da PA.

Perante a evidência disponível não é possível determinar o valor alvo ideal de PA em doentes diabéticos. Porém, este estudo reforça a importância do controlo adequado da PA nestes doentes na prática clínica, uma vez que a presença simultânea de DM e de HTA duplica o risco de doença cardiovascular e aumenta o risco de complicações macro e microvasculares. ${ }^{28}$ Desta forma, é necessária uma abordagem multifatorial dos fatores de risco cardiovasculares, bem como uma avaliação cuidadosa e individualizada dos riscos e benefícios que possam decorrer de uma estratégia de tratamento mais intensivo da PA.

\section{REFERÊNCIAS BIBLIOGRÁFICAS}

1. World Health Organization. Global report on diabetes. Geneva: WHO; 2016. ISBN 9789241565257

2. Stamler J, Vaccaro O, Neaton JD, Wentworth D. Diabetes, other risk factors, and 12-yr cardiovascular mortality for men screened in the Multiple Risk Factor Intervention Trial. Diabetes Care. 1993;16(2):434-44.

3. Sarwar N, Gao P, Seshasai SR, Gobin R, Kaptoge S, Di Angelantonio E, et al. Diabetes mellitus, fasting blood glucose concentration, and risk of vascular disease: a collaborative meta-analysis of 102 prospective studies. Lancet. 2010;375(9733):2215-22.

4. Penno G, Solini A, Bonora E, Fondelli C, Orsi E, Zerbini G, et al. HbA1c variability as an independent correlate of nephropathy, but not retinopathy, in patients with type 2 diabetes: the Renal Insufficiency And Cardiovascular Events (RIACE) Italian multicenter study. Diabetes Care. 2013;36(8):2301-10

5. Patel A, MacMahon S, Chalmers J, Neal B, Woodward M, Billot L, et al. Effects of a fixed combination of perindopril and indapamide on macrovascular and microvascular outcomes in patients with type 2 diabetes mellitus (the ADVANCE trial): a randomised controlled trial. Lancet. 2007;370(9590):829-40.

6. Deedwania PC. Diabetes and hypertension, the deadly duet: importance, therapeutic strategy, and selection of drug therapy. Cardiol Clin. 2005;23(2):139-52.

7. UK Prospective Diabetes Study Group. Tight blood pressure control and risk of macrovascular and microvascular complications in type 2 diabetes: UKPDS 38. BMJ. 1998;317(7160):703-13.

8. Heart Outcomes Prevention Evaluation Study Investigators. Effects of ramipril on cardiovascular and microvascular outcomes in people with diabetes mellitus: results of the HOPE study and MICRO-HOPE substudy. Lancet. 2000;355(9200):253-9.

9. Chobanian AV, Bakris GL, Black HR, Cushman WC, Green LA, Izzo JL Jr, et al. The seventh report of the Joint National Committee on prevention, detection, evaluation, and treatment of high blood pressure: the JNC 7 report. JAMA. 2003;289(19):2560-72.

10. Mancia G, De Backer G, Dominiczak A, Cifkova R, Fagard R, Germano G, 
et al. 2007 Guidelines for the management of arterial hypertension:The Task Force for the management of arterial hypertension of the European Society of Hypertension (ESH) and of the European Society of Cardiology (ESC). Eur Heart J. 2007;28(12):1462-536.

11. Buse JB, Ginsberg HN, Bakris GL, Clark NG, Costa F, Eckel R, et al. Primary prevention of cardiovascular diseases in people with diabetes mellitus: a scientific statement from the American Heart Association and the American Diabetes Association. Circulation. 2007;115(1):114-26.

12. Brunström M, Carlberg B. Effect of antihypertensive treatment at different blood pressure levels in patients with diabetes mellitus: systematic review and meta-analyses. BMJ. 2016;352:i717.

13. Bangalore S, Kumar S, Lobach I, Messerli FH. Blood pressure targets in subjects with type 2 diabetes mellitus/impaired fasting glucose: observations from traditional and bayesian random-effects meta-analyses of randomized trials. Circulation. 2011;123(24):2799-810.

14. Institute for Clinical Systems Improvement. Diagnosis and management of type 2 diabetes mellitus in adults [homepage]. ICSI; 2014. Available from: https://www.icsi.org/guidelines_more/catalog_guidelines_and_ more/catalog_guidelines/catalog_endocrine_guidelines/diabetes/

15. James PA, Oparil S, Carter BL, Cushman WC, Dennison-Himmelfarb C, Handler J, et al. 2014 Evidence-based guideline for the management of high blood pressure in adults: report from the panel members appointed to the Eighth Joint National Committee (JNC 8). JAMA. 2014; 311(5):507-20.

16. National Institute for Health and Care Excellence. Type 2 diabetes in adults: management [homepage]. London: NICE; 2015 Dec [updated 2017 May]. Available from: https://www.nice.org.uk/guidance/ng28

17. Franklin SS, Larson MG, Khan SA, Wong ND, Leip EP, Kannel WB, et al. Does the relation of blood pressure to coronary heart disease risk change with aging? The Framingham Heart Study. Circulation. 2001;103(9):1245-9.

18. Emdin CA, Rahimi K, Neal B, Callender T, Perkovic V, Patel A. Blood pressure lowering in type 2 diabetes: a systematic review and meta-analysis. JAMA. 2015;313(6):603-15.

19. Ismail-Beigi F, Craven TE, O'Connor PJ, Karl D, Calles-Escandon J, Hramiak I, et al. Combined intensive blood pressure and glycemic control does not produce an additive benefit on microvascular outcomes in type 2 diabetic patients. Kidney Int. 2012;81(6):586-94.

20. American Diabetes Association. Cardiovascular disease and risk management. Diabetes Care. 2016;39(Suppl 1):S60-S71.

21. Mancia G, Fagard R, Narkiewicz K, Redon J, Zanchetti A, Böhm M, et al.
2013 ESH/ESC guidelines for the management of arterial hypertension: the Task Force for the Management of Arterial Hypertension of the European Society of Hypertension (ESH) and of the European Society of Cardiology (ESC). Eur Heart J. 2013;34(28):2159-219.

22. Hackam DG, Khan NA, Hemmelgarn BR, Rabkin SW, Touyz RM, Campbell NR, et al. The 2010 Canadian Hypertension Education Program recommendations for the management of hypertension: part 2 - therapy. Can J Cardiol. 2010;26(5):249-58.

23. Working group of the Clinical Practice Guideline for type 2 Diabetes. Clinical practice guideline on type 2 diabetes [Internet]. Madrid: Health Technologies Assessment Agency of the Basque Country; 2008. Available from: http://www.guiasalud.es/GPC/GPC_429_Diabetes_2_Osteba_compl_en.pdf

24. Rotaeche del Campo R, Aguirrezabala Jaca J, Balagué Gea L, Gorroñogoitia Iturbe A, Idarreta Mendiola I, Mariñelarena Mañeru E, et al. Clinical practice guidelines on arterial hypertension: 2007 update. Vitoria-Gasteiz: Osakidetza; 2008. ISBN 9788469130681

25. Cushman WC, Evans GW, Byington RP, Goff DC Jr, Grimm RH Jr, Cutler JA, et al. Effects of intensive blood-pressure control in type 2 diabetes mellitus. N Engl J Med. 2010;362(17):1575-85.

26. Rabi DM, Padwal R, Tobe SW, Gilbert RE, Leiter LA, Quinn RR, et al. Risks and benefits of intensive blood pressure lowering in patients with type 2 diabetes. CMAJ. 2013;185(11):963-7.

27. Weir MR, Yeh F, Silverman A, Devereux RB, Galloway JM, Henderson JA, et al. Safety and feasibility of achieving lower systolic blood pressure goals in persons with type 2 diabetes: the SANDS trial. J Clin Hypertens (Greenwich). 2009;11(10):540-8.

28. Reboldi G, Gentile G, Manfreda VM, Angeli F, Verdecchia P. Tight blood pressure control in diabetes: evidence-based review of treatment targets in patients with diabetes. Curr Cardiol Rep. 2012;14(1):89-96.

\section{CONFLITO DE INTERESSES}

As autoras declaram não possuir quaisquer conflitos de interesse.

\section{ENDEREÇO PARA CORRESPONDÊNCIA}

Vânia Gomes

E-mail: vania.612@hotmail.com

http://orcid.org/0000-0003-1507-5657

\section{Recebido em 28-09-2016}

Aceite para publicação em 04-07-2018 


\section{ABSTRACT}

\section{INTENSIVE BLOOD PRESSURE CONTROL IN TYPE 2 DIABETES MELLITUS: WHAT IS THE EVIDENCE?}

Aim: To verify if intensive antihypertensive treatment (systolic blood pressure $<130 \mathrm{mmHg}$ ) has beneficial effects on cardiovascular morbidity and mortality in patients with type 2 diabetes mellitus and hypertension, compared with standard antihypertensive treatment (systolic blood pressure $<140 \mathrm{mmHg}$ ).

Data sources: National Guideline Clearinghouse, NHS Evidence, CMA InfoBase, Cochrane, DARE, MEDLINE/PubMed.

Review methods: Meta-analyses (MA), systematic reviews, randomized controlled trials (RCT) and guidelines in English and Portuguese, published between 2006 and 2016 were searched, using the MeSH terms 'antihypertensive agents', 'diabetes mellitus', and 'blood pressure'. The Strength of Recommendation Taxonomy (SORT) scale from the American Family Physician was used to evaluate the levels of evidence (LE) and the strength of recommendation (SR).

Results: A total of 662 articles were obtained, amongst which 12 fulfilled the inclusion criteria: three MA, one RCT and eight guidelines. MA showed that intensive blood pressure (BP) control reduces the risk of stroke, microalbuminuria and nephropathy in diabetic patients (LE 2). However, intensive BP control was associated with an increased risk of adverse effects in these patients, and seems to increase cardiovascular mortality (LE 2). The RCT has only shown a reduction on the risk of development of microalbuminuria (LE 2). Finally, most guidelines do not recommend intensive BP control in diabetic patients and advise that the BP control should be more intensive only in young diabetic patients, in those with target organ damage, or those with more than one risk factor for atherosclerotic cardiovascular disease.

Conclusions: According to the evidence available, intensive BP control in patients with type 2 diabetes should not be recommended, except in young diabetic patients and in certain risk groups (SR B). Nevertheless, this study reinforces the importance of BP control in these patients, and highlights the key role of the family physician in the individualized assessment of the risks and benefits of intensive BP treatment.

Keywords: Hypertension; Diabetes mellitus, Type 2; Antihypertensive agents. 